\title{
Relations entre l'état hydrique du sol, le potentiel de base et d'autres indicateurs de la contrainte hydrique chez le maïs.
}

\author{
F Tardieu 1, N Katerji 2, O Bethenod 2 \\ avec la collaboration technique de $\mathrm{P}$ Hamard, $\mathrm{P}$ Quétin et $\mathrm{P}$ Bal \\ ${ }^{1}$ INRA, Agronomie; \\ ${ }^{2}$ INRA, Bioclimatologie F-7850 Thiverval-Grigon, France
}

(Reçu le 4 avril 1990; accepté le 15 août 1990)

\begin{abstract}
Résumé - Des indicateurs de l'état hydrique du sol (potentiel hydrique dans la zone racinaire et réserve en eau utilisée) ont été mis en relation avec plusieurs indicateurs de l'état hydrique de plantes de maïs pendant la période postfloraison : le potentiel foliaire au lever du jour, le potentiel foliaire pendant la journée, la conductance stomatique et le niveau des composantes du rendement formées pendant la période de mesure. Cette mise en relation a été effectuée pour des plantes en pots, où l'état hydrique du sol et la réserve en eau sont bien définis, et, pendant 3 années, pour des plantes au champ, où les grandeurs caractérisant l'état hydrique du sol sont fortement variables spatialement. Dans les 2 cas, on a suivi l'état hydrique des plantes pour des proportions de la réserve en eau du sol utilisée allant de 15 à $100 \%$ de la «réserve utile». Dans l'essai en pots, on a observé le cas classique pour le maïs : le potentiel de base et la conductance stomatique ont décru avec le potentiel hydrique du sol, alors que le potentiel foliaire minimal mesuré pendant la journée a peu varié. Dans l'essai au champ, le potentiel de base et la conductance stomatique sont restés stables sur l'ensemble de la gamme de variation de la réserve en eau du sol, et l'épuisement de la réserve utile estimée du sol n'a pas affecté les composantes du rendement élaborées pendant la période de mesures. Les plantes ne se sont donc pas trouvées en stress hydrique, même lorsque la réserve utile estimée du sol était épuisée. Ceci ne peut être expliqué que par une forte contribution des couches de sol non enracinées à l'alimentation hydrique des plantes. Les ordres de grandeur de flux d'eau à travers la profondeur d'enracinement, calculés à partir des gradients observés de potentiel hydrique et de la conductivité hydraulique calculée du sol, sont compatibles avec cette hypothèse. Ces observations peuvent avoir des incidences importantes sur la gestion de l'irrigation et sur le diagnostic de contraintes hydriques au champ.
\end{abstract}

Zea mays L / potentiel foliaire / potentiel de base / conductance stomatique / composantes du rendement / potentiel du sol / bilan hydrique

Summary - Relationship between soil water status, predawn leaf water potential and other indicators of the plant water status in maize. Indicators of soil water status (water potential in the root zone and soil water reserve) have been related to several indicators of the plant water status of maize after silking. The latter were the predawn leaf water potential, the daytime leaf water potential, the stomatal conductance and the measurement of yield components formed during the post-silking period. This relationship was established for plants in pots, where soil water status and water reserve are well defined, and in the field, where the soil water status has a high spatial variability. The field experiment was repeated for 3 years with contrasting climate. Yield components were only measured in the field experiment. Soil water reserve in the field was calculated using measured rooting depths (fig 2) and soil water release curves corresponding to each soil layer. In both cases, water status of plants was measured for water reserves in the soil ranging from 15-100\% of the total water reserve (figs 1 and 3). In the pot experiment, we observed the classical case for maize: predawn leaf water potential and stomatal conductance decreased with the soil water potential, but daytime leaf water potential remained almost constant during the experiment (figs $5 \mathrm{a}$ and $6 \mathrm{a}$ ). In the field stomatal conductance and predawn leaf water potential did not vary over the whole range of soil water reserve and depleting the latter in 1989 did not affect the yield components (figs $5 \mathrm{~b}$ and $6 \mathrm{~b}$; tab II). Thus, plants did not experience water stress, even when the calculated soil water reserve had been depleted. This can be explained only by an appreciable contribution of non-rooted layers to water uptake by plants. The order of magnitude of water flux through the rooting depth, calculated at each date using the observed gradient of soil water potential (fig 4) and calculated soil hydraulic conductivity at the rooting depth, are consistent with this hypothesis. These observations may have appreciable consequences for irrigation warning and for diagnosis of water stress in the field. 


\section{INTRODUCTION}

Le diagnostic de l'état hydrique des plantes peut avoir pour objectif, soit le pilotage de l'irrigation, il doit alors être mené en temps réel, soit l'analyse de chutes de rendement ou des conséquences de techniques culturales, il est alors souvent effectué a posteriori. Ces 2 objectifs peuvent être remplis par des modèles d'extraction d'eau par les plantes (cf les synthèses bibliographiques de Moltz, 1981 et de Feddes et al, 1988). Ces modèles sont cependant difficiles à mettre en œuvre à cause du grand nombre de variables d'entrée qui leur est nécessaire, ainsi que de la difficulté pour déterminer expérimentalement certaines de ces variables (les caractéristiques du système racinaire et la conductivité hydraulique du sol, en particulier). En outre, les modèles actuels de transfert d'eau sont mal adaptés à l'étude des conséquences de techniques culturales (Tardieu, 1989). C'est pourquoi le diagnostic est encore, en général, fondé sur des indicateurs, certains n'ayant de sens qu'en temps réel, d'autres pouvant être déterminés a posteriori.

- Un premier type d'indicateurs est fondé sur la caractérisation de l'état hydrique du sol. Le peuplement végétal est considéré comme étant à l'état optimal et comme ayant une évapotranspiration maximale jusqu'à une valeur seuil de réserve d'eau du sol (réserve facilement utilisable, RFU), égale à une fraction de la réserve utile (RU) (Eagleman, 1971; Choisnel, 1985).

- Une seconde approche pour obtenir des indicateurs consiste à caractériser l'état hydrique des plantes (Katerji et al, 1988; Jones, 1980), soit directement par le potentiel ou la teneur en eau des feuilles, soit indirectement par la conductance stomatique qui est en relation étroite avec l'assimilation nette, la température de surface du couvert (Jackson, 1982) ou les variations morphologiques de certains organes (Klepper et al, 1971; Huguet, 1985). Le potentiel hydrique des feuilles mesuré en fin de nuit (potentiel de base) est intermédiaire entre les indicateurs "sol» et "plante». Mesuré après une période de plusieurs heures sans transpiration importante, il représente un état d'équilibre entre l'état hydrique de la plante et celui du sol. II peut alors être considéré comme une mesure intégrée de ce dernier.

Lorsque les espèces considérées ont une floraison de courte durée, un indicateur supplémentaire peut être le niveau de la composante du rendement déterminée au moment d'une contrainte hydrique supposée (Robelin, 1963; Claasen et Shaw, 1970).

Nous avons cherché à mettre en relation ces 3 types d'indicateurs, d'une part pour tester certaines des hypothèses sur lesquelles ils sont fondés, d'autre part pour contribuer à étudier leur domaine de validité. Les questions posées étaient, en particulier, la relation entre le potentiel de base et le potentiel du sol, la validité du calcul d'une réserve facilement utilisable, et la cohérence entre les indicateurs caractérisant l'état hydrique du sol et ceux caractérisant la plante Cette mise en relation a été effectuée pour le maiis à la floraison, dans 2 conditions :

- pour des plantes en pots où le potentiel hydrique du sol, peu variable spatialement, peut être synthétisé par un potentiel moyen, et où la réserve d'eau est définie et mesurable parce que le système a une limite inférieure imperméable (système semi-fermé),

- au champ, où le potentiel du sol ne peut être caractérisé que par une fonction de répartition et où la profondeur sur laquelle la réserve d'eau est calculée est mal définie (système ouvert). Nous avons bénéficié de 3 années climatiques contrastées, ce qui a permis d'effectuer ce travail pour une large gamme d'états hydriques du sol.

\section{MATÉRIELS ET MÉTHODES}

\section{Essai en pots}

Le maïs a été semé le 14 avril 1989 dans 220 pots de 5 I remplis d'un mélange de tourbe et de perlite. Quatre de ces pots étaient situés sur une balance à 1 $\mathrm{g}$ de précision reliée à un micro-ordinateur, afin que l'extraction d'eau du sol puisse être suivie. Le potentiel hydrique du sol a été mesuré en continu à l'aide de 8 psychromètres reliés à un microvoltmètre à acquisition de données (Wescor 11c), placé dans une enceinte isotherme. Les données des mêmes psychromètres étaient vérifiées 2 fois par jour avec un microvoltmètre manuel (Wescor 33c). Huit tensiomètres ont été placés dans les pots afin de suivre le potentiel dans la gamme $-0,01$ à $-0,08 \mathrm{MPa}$. Cependant, ceux-ci n'ont pas réagi à la baisse de teneur en eau du sol, probablement à cause d'un mauvais contact entre la bougie et le substrat que nous avions choisi. L'irrigation automatique a été stoppée sur la moitié des pots le 18 juin, 2 jours après le début de la floraison. La réserve en eau de ces pots a été évaluée directement en mesurant la teneur en eau au début de l'expérience, où les pots étaient à la capacité de rétention, et à la mort des plantes. Elle s'élevait à 1,3 I par pot.

Le potentiel hydrique foliaire a été mesuré à la fin de la nuit (potentiel de base), et à $12 \mathrm{~h}$ (heure solaire). 
Six feuilles ont été prélevées et insérées dans une chambre à pression. La conductance stomatique des faces supérieures et inférieures de 10 feuilles a été mesurée à $9,11,12$ et $13 \mathrm{~h}$ en utilisant un poromètre à diffusion (Delta $\mathrm{T}$ III). Ces mesures ont été réalisées sur la feuille de l'épi, en ne retenant que des feuilles recevant la lumière directe. Les résultats présentés sont les moyennes des conductances des faces inférieures et supérieures des feuilles. L'étalonnage du poromètre a été réalisé après chaque série de mesures. La conductance stomatique $\left(g_{S}\right)$ a été reliée aux mesures du poromètre ( $t$ ) par régression non linéaire sur une hyperbole

$$
g_{s}=\frac{a t+b}{t+c}
$$

Le rayonnement PAR a été mesuré 4 fois par heure pendant les périodes d'étude avec un quantum sensor (Licor Li 190S).

\section{Essai au champ}

L'essai a été réalisé de 1987 à 1989 à Grignon (Région parisienne) sur une parcelle de sol argilolimoneux profond, dont les caractéristiques sont présentées au Tableau I. La masse volumique du sol a été mesurée avec un densimètre à atténuation de rayons gamma (LPC-INRA, distance entre pals : 30 $\mathrm{cm}$ ). Le maïs (hybride simple LG1) a été semé le 5 mai, 30 avril et 2 mai, respectivement, en 1987, 1988 et 1989 , à une densité de 8,9 plantes. $\mathrm{m}^{-2}$. La floraison a été atteinte les 30,19 et 15 juillet, respectivement. Les indices de surface foliaire (LAl) étaient de 2,97, 3,0 et 2,8 à cette date. L'enracinement a été caractérisé en 1988 par mesure de la longueur de racines par horizon. Vingt-quatre carottes de sol ont été prélevées entre 0 et $1,25 \mathrm{~m}$. Les racines ont été séparées du sol avec une machine hydropneumatique (Smucker et al, 1982), et leur longueur a été mesurée à l'aide d'un équipement vidéo ( $\Delta T$ area meter). En 1989, 6 cartes racinaires de $(0,80 \times 1,25) \mathrm{m}$ ont été réalisées sur des plans verticaux, avec une maille de $20 \mathrm{~mm}$ (Tardieu et Manichon, 1986).

Six tubes d'accès ont été placés chaque année à $0,1 \mathrm{~m}$ du rang de semis. Les variations au cours du temps de la teneur en eau volumique du sol ont été suivies en utilisant un humidimètre neutronique (Nardeux solo $25,1.48 \mathrm{GBq}$ ) entre 0,20 et $1,75 \mathrm{~m}$ de profondeur. De 5 à 6 mesures par tube ont été réalisées tous les $0,1 \mathrm{~m}$ à chaque date. Des échantillons de terre ont été prélevés tous les $0,1 \mathrm{~m}$ avant la pose des tubes, et une mesure neutronique a été effectuée immédiatement après la pose. Cette opération a été répétée à la fin de chaque saison de mesures. Un ajustement linéaire entre comptages et teneur en eau a été effectué, avec une pente unique pour chaque horizon de $0,1 \mathrm{~m}$, mais une ordonnée à l'origine particulière pour chaque profondeur de chaque tube. La teneur en eau de la couche entre 0 et $0,2 \mathrm{~m}$ a été mesurée périodiquement par méthode gravimétrique dans les couches $0-30,30-90,90-200 \mathrm{~mm}$.

Six séries de tensiomètres ont été placées dans le sol tous les $0,20 \mathrm{~m}$ jusqu'à $1,20 \mathrm{~m}$, et 2 séries à 1,50 , 1,70 et $1,90 \mathrm{~m}$ de profondeur. Les potentiels hydriques inférieurs à $-0,08 \mathrm{MPa}$ ont été calculés à partir de la teneur en eau du sol et de la relation Potentiel Teneur en eau de la couche correspondante. Cette relation a été obtenue in situ pour les potentiels hydriques supérieurs à $-0,08 \mathrm{MPa}$ en combinant mesures tensiométriques et gravimétriques sur les mêmes sites, et au laboratoire pour la gamme de potentiels entre $-1,6$ et $-0,01 \mathrm{MPa}$. Elle a été ajustée dans la gamme entre $-0,01 \mathrm{MPa}$ et $-0,3 \mathrm{MPa}$ sur l'expression analytique proposée par Van Genuchten (1980)

$$
\frac{\theta-\theta_{r}}{\theta_{s}-\theta_{r}}=\left[1 /\left(1+(\alpha \psi)^{m}\right)\right]^{m}
$$

où $m=1-(1 / n)$, et où $n, \alpha, \theta_{s}$ et $\theta_{r}$ sont des paramètres ajustés. Cet ajustement étant insatisfaisant pour les potentiels inférieurs à $-0,3 \mathrm{MPa}$, un ajuste-

Tableau I. Caractéristiques de la parcelle étudiée. $W_{c c}:$ teneur en eau de la capacité au champ; $W_{1,6}:$ teneur en eau à 1,6 MPa, $\rho$ : masse volumique moyenne de l'horizon.

\begin{tabular}{lccc}
\hline & Couche labourée & Horizon A & Loess \\
\hline Profondeurs $(m)$ & $0-0,30$ & $0,30-0,80$ & $\begin{array}{c}0,80-2 \\
\text { (sur calcaire grossier) }\end{array}$ \\
Calcaire & & & 28 \\
total $(\%)$ & 0,9 & 0,2 & 17 \\
Argile $(\%)$ & 18 & 15 & 4 \\
Sable $(\%)$ & 7 & 4 & 0,20 \\
$W_{\mathrm{cc}}\left(g \cdot g^{-1}\right)$ & 0,22 & 0,20 & 0,10 \\
$W_{1,6}\left(g \cdot g^{-1}\right)$ & 0,12 & 0,12 & 1,44 \\
$\rho\left(g \cdot m^{-3}\right)$ & 1,35 & 1,43 & \\
\hline
\end{tabular}


ment linéaire reliant $\log (-\psi)$ à $\theta$ a été adopté pour cette gamme. Le bilan hydrique du sol a été calculé en mesurant les termes de la relation

$$
P-E T R-[\Delta S]^{z}+D_{z}+R=0
$$

où $P$ est la valeur des précipitations, ETR l'évapotranspiration réelle, $[\Delta S]^{z}$ la variation de stock d'eau du sol entre la surface et une profondeur $z, D_{z}$ le transfert d'eau (montant ou descendant) à travers cette profondeur $z$, et $R$ le ruissellement. Ce dernier terme a été négligé, étant donné la faible pente de la parcelle et les valeurs élevées de conductivité hydraulique saturée du sol $\left(10^{-6} \mathrm{~m} \cdot \mathrm{s}^{-1}\right)$. Contrairement à l'étude effectuée par Katerji et al, (1984), l'ETR n'a pas été mesurée directement par la méthode du bilan d'énergie. Nous en avons estimé les valeurs maximales (ETM) à l'aide du calcul de l'évapotranspiration potentielle journalière (ETP) par la formule de Penman. Étant donné les surfaces foliaires mesurées chaque année et la gamme d'ETP journalières observées $(0-5 \mathrm{~mm})$, nous avons considéré une ETM égale à l'ETP. Ceci est acceptable compte tenu des vérifications expérimentales réalisées dans la région sur des maïs irrigués avec des évapotranspiromètres (Perrier et al, 1974) ou par la méthode du rapport de Bowen (Katerji, non publié). La variation de stock d'eau du sol $\left([\Delta S]^{7}\right)$ a été calculée pour chaque date entre la surface du sol et une profondeur $z$ située, pour chaque date, au plan de flux nul déterminé à partir du suivi tensiométrique. De cette façon, le terme $D_{z}$ était nul à la profondeur $z$. Lorsque ce plan n'a plus été observable, la profondeur considérée était celle des tubes $(1,75 \mathrm{~m})$, et un terme $D_{z}$ a dû être considéré. La date de référence pour ce calcul était, chaque année, la dernière date avant floraison où la teneur en eau du sol correspondait à la "capacité au champ". La variation de bilan hydrique liée à la distance au rang de semis a été négligée, comme le permettait une étude préalable (Tardieu, 1988a) menée sur la même variété dans le même site. Le flux d'eau à travers la profondeur $(z)$ sur laquelle le bilan hydrique était réalisé à été calculé par la loi de Darcy :

$$
D_{z}=-K(\theta)(\mathrm{d} \psi / \mathrm{d} z-1)
$$

où $K(\theta)$ est la conductivité hydraulique à la profondeur $z$ et $\mathrm{d} \psi / \mathrm{d} z$ est le gradient de potentiel du sol autour de cette profondeur. La conductivité hydraulique a été évaluée par des mesures de laboratoire (Bruckler et al, 1991) et ajustée sur le modèle de Mualem (1976) modifié par Van Genuchten (1980).

Le potentiel hydrique foliaire et la conductance stomatique ont été mesurés approximativement toutes les heures entre 8 et 15 h, sur 10.à 15 (suivant la variabilité des données) feuilles de l'épi recevant un éclairement direct. La conductance a été mesurée avec une chambre de Parkinson en circuit ouvert $(A D C)$ chaque année, et un poromètre à diffusion ( $\triangle T$ III) en 1987 et 1989. La correspondance entre ces 2 appareils s'est révélée satisfaisante, les données présentées sont celles de la chambre de Parkinson pour 1987 et 1988, et du poromètre pour 1989. L'étalon- nage du poromètre a été effectué d'une manière similaire à celle décrite pour l'essai en serre; celui de la chambre de Parkinson a été mené par la méthode décrite par Bethenod et al (1988). Le rayonnement PAR a été mesuré de la même façon que dans l'essai en serre. Les mesures ont été effectuées pendant les 2 semaines suivant la floraison, période pendant laquelle la conductance stomatique du maïs varie peu avec le stade végétatif (Bethenod et al, 1982).

Nous n'avons retenu que des journées situées pendant des périodes sans pluies où l'éclairement était supérieur à $1 \mathrm{mmol}$ (quanta) $\cdot \mathrm{m}^{-2} \cdot \mathrm{s}^{-1}$.

La récolte a été effectuée au champ chaque année après l'apparition de la couche noire (Daynard et Duncan, 1969) sur 10 placettes de $9,6 \mathrm{~m}^{2}$ avec 8 plantes chacune. Le nombre de grains et le poids de grains ont été mesurés sur chaque placette. Cette variable n'a pas pu être mesurée dans l'essai en pots, étant donné que les plantes ont atteint le point de flétrissement permanent à la fin de l'essai.

\section{RÉSULTATS}

\section{Essai en pots}

Le dessèchement du sol dans les pots a été rapide pendant les 4 premières journées suivant l'arrêt de l'irrigation, atteignant le 21 juin, $68 \%$ de la réserve maximale (fig $1 \mathrm{a}$ ). II a atteint chaque jour un maximum à $12 \mathrm{~h}$ (heure solaire). Le potentiel hydrique du sol $\left(\psi_{S}\right)$ mesuré par psychrométrie était similaire pour les 2 types de voltmètres utilisés, manuel et automatique (fig 1b). II
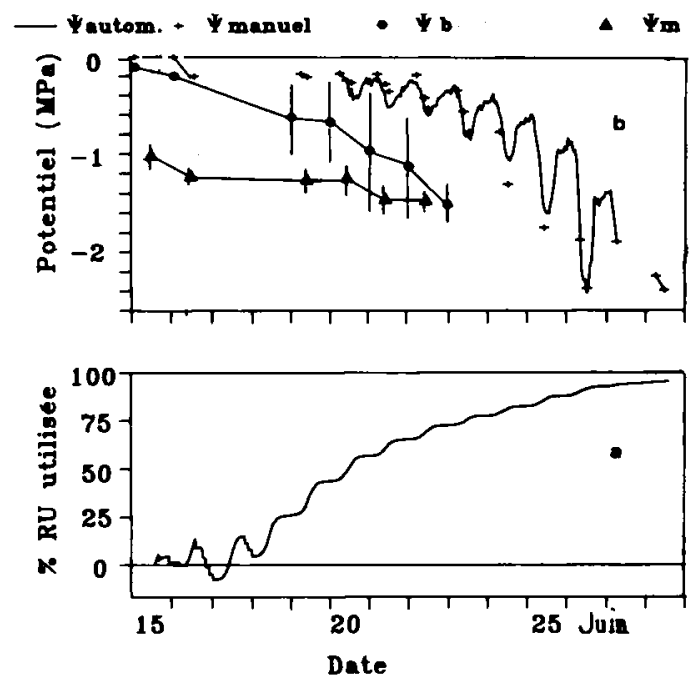

Fig 1. Variations dans le temps de la réserve en eau du sol dans l'essai en pots (a), et des indicateurs de l'état hydrique du sol et des plantes (b) : potentiel du sol mesuré avec un psychromètre manuel $\left(\psi_{\text {manuel }}\right)$ ou à acquisition de données ( $\left.\psi_{\text {autom }}\right)$; potentiel foliaire de base $\left(\psi_{b}\right)$; potentiel foliaire à midi solaire $\left(\psi_{m}\right)$. 
a subi des variations journalières s'amplifiant avec le temps, le maximum se situant approximativement à $4 \mathrm{~h}$ et le minimum à $14 \mathrm{~h}$, respectivement. Les valeurs de $\psi_{s}$ mesurées pendant la journée sont devenues inférieures à $-1,6 \mathrm{MPa}$ à partir du 25 juin, les valeurs maximales journalières ont franchi ce seuil le 27 juin. Ces variations journalières de $\psi_{s}$ sont probablement dues au fait que les psychromètres mesurent le potentiel de l'atmosphère du sol, qui n'est pas à l'équilibre avec la totalité de la phase liquide pendant la journée. II est également possible que les variations de température aient eu un effet, bien que les variations mesurées de $\psi_{s}$ n'aient pas été en phase avec celles de la température comme l'avait déjà remarqué Bruckler (1984). Le potentiel hydrique de base $\left(\psi_{b}\right)$ a décru de $-0,2$ à $-1,5 \mathrm{MPa}$, entre le début et la fin de l'expérience (fig 1), avec des écarts types croissants lorsque le potentiel moyen baissait. II était systématiquement inférieur de 0,4 à $0,6 \mathrm{MPa}$ au potentiel du sol mesuré aux mêmes heures. Le potentiel foliaire minimal, mesuré pendant la journée, a décru de $-1,0$ à $-1,5 \mathrm{MPa}$ pendant la même période.

\section{Essai au champ}

La profondeur d'enracinement au champ était approximativement de $1,10 \mathrm{~m}$ en 1988 et 1989 (fig 2). La majeure partie des racines a été bloquée à $0,80 \mathrm{~m}$ par une couche de loess calcaire. Seules quelques racines non ramifiées ont pénétré ce loess à travers des galeries de vers de terre. On peut noter que la méthode de carottage a fourni une profondeur inférieure à celle évaluée par cartographie, à cause du moindre taux d'échantillonnage. La profondeur de 1,10 m correspond à une réserve utile estimée $(R U)$ de $135 \mathrm{~mm}$.

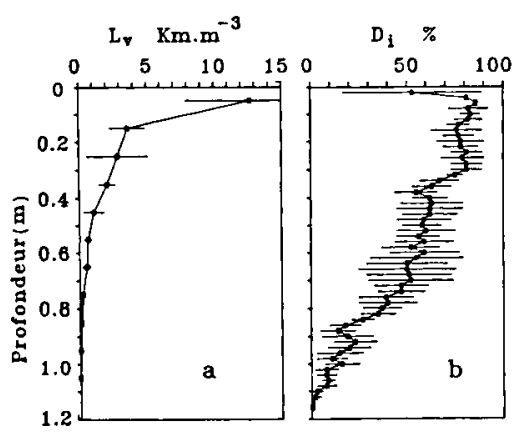

Fig 2. Relation entre la densité racinaire et la profondeur. (a) 1988 : Iongueur de racines par unité de volume $(L v)$. (b) 1989 : densités d'impacts racinaires déterminées sur cartes racinaires (Di), en proportion de cases de $2 \times 2 \mathrm{~cm}$ avec au moins un impact de racine. Barres horizontales : intervalle de confiance $p=0,05$.
La figure 3 présente, pour chaque année étudiée, la variation de stock d'eau du sol $\left([\Delta S]^{2}\right)$ entre la surface et le plan de flux nul, et le bilan $E T P$ - $P$ calculé pour la même période. En 1987, une première période de dessèchement préfloraison a été interrompue par des précipitations de $86 \mathrm{~mm}$ entre le 13 et le 21 juillet. Elle a été suivie par une seconde période où $[\Delta S]^{z}$ a atteint $82 \mathrm{~mm}$. Trois périodes sans pluie plus courtes ont correspondu à des variations de réserve d'eau inférieures à $60 \mathrm{~mm}$ en 1988 . En 1989, $[\Delta S]^{z}$ a atteint des valeurs proches de la $R U$ estimée à partir de la fin du mois de juillet, et a dépassé celle-ci lors de la dernière mesure réalisée en septembre. Lors des 3 années, les variations de stock d'eau du sol ont suivi étroitement le terme ETP - $P$ calculé sur les mêmes périodes, sauf à partir de la mi-juillet 1989 , lorsque $[\Delta S]^{z}$ s'est rapproché de la $R U$ estimée.

Ce dépassement de la réserve utile n'a pas été causé en premier lieu par le dépassement de la limite de $-1,6 \mathrm{MPa}$ pour $\psi_{s}$, qui ne s'est produit que dans les couches superficielles (fig 4 ), et qui correspond à une faible variation de volume d'eau. Il est dû à une baisse de potentiel au-delà de la profondeur d'enracinement. La variation de réserve d'eau du sol entre la profondeur d'enracinement et $1,75 \mathrm{~m}$ a été de $35 \mathrm{~mm}$ entre le 8 juin et le 12 septembre. Cependant, la figure 4 montre que cette couche subissait probablement un transfert d'eau en régime permanent, étant donné le gradient de potentiel qui y existait. Une évaluation du flux d'eau à travers la

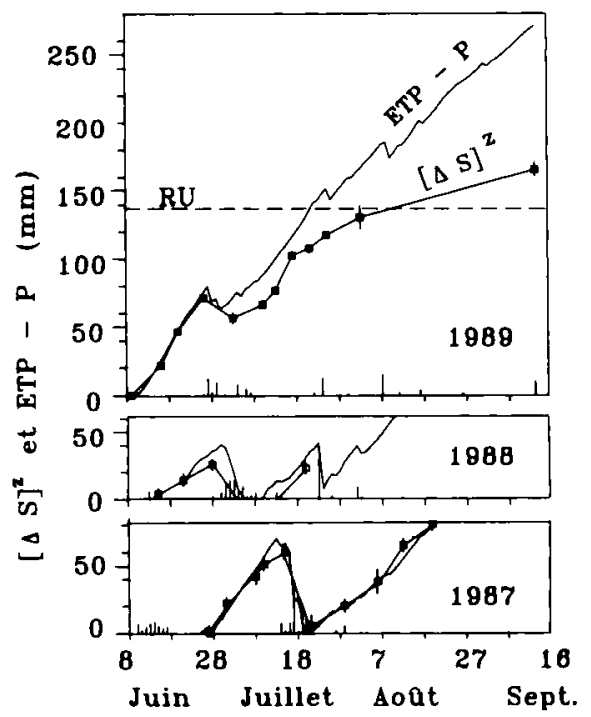

Fig 3. Variations de la réserve en eau du sol $\left([\Delta S]^{2}\right)$ entre la surface et la profondeur du plan de flux nul (jusqu'à ce que celle-ci soit supérieure à $1,75 \mathrm{~m}$ ), et calcul du bilan ETP $-P$ en 1987, 1988 et 1989. Les barres verticales à partir de l'axe des abscisses représentent les précipitations. $R U$ : réserve utile calculée entre la surface et la profondeur d'enracinement $(1,10 \mathrm{~m})$. Barres verticales autour des moyennes : intervalle de confiance $p=0,05$. 


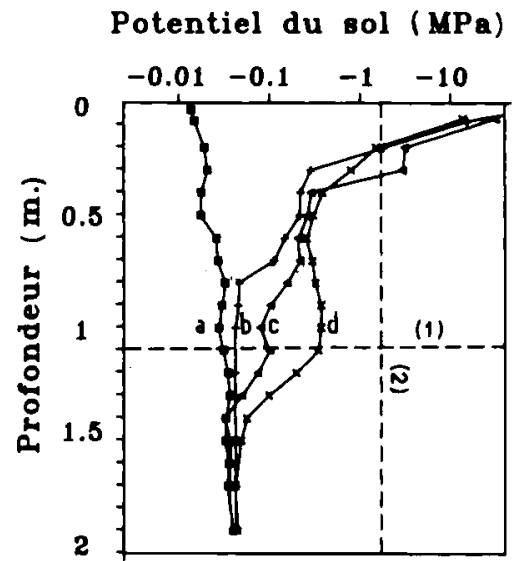

Fig 4. Potentiels hydriques successifs du sol les 9 juin (a), 18 juillet (b), 2 aoút (c) et 12 septembre (d). (1) : profondeur d'enracinement; (2) $-1,6 \mathrm{MPa}$.

profondeur d'enracinement a été calculée à partir de l'équation (4). Elle aboutit à un terme $D_{z}$ de l'ordre de $1 \mathrm{~mm} \cdot \mathrm{j}^{-1}$ à partir de fin juillet. La prudence s'impose cependant par rapport à cette évaluation, en raison de la grande incertitude existant sur l'évaluation de la conductivité hydraulique.

\section{Relation entre la réserve d'eau du sol et l'état hydrique des plantes}

Les figures 5 et 6 présentent les valeurs de potentiel hydrique foliaire minimal journalier $\left(\psi_{m}\right)$, de potentiel de base $\left(\psi_{b}\right)$, et de conductance stomatique $\left(g_{s}\right)$ maximale journalière, en fonction de la proportion de réserve d'eau extraite du sol calculée par rapport à la $R U$ estimée. Dans

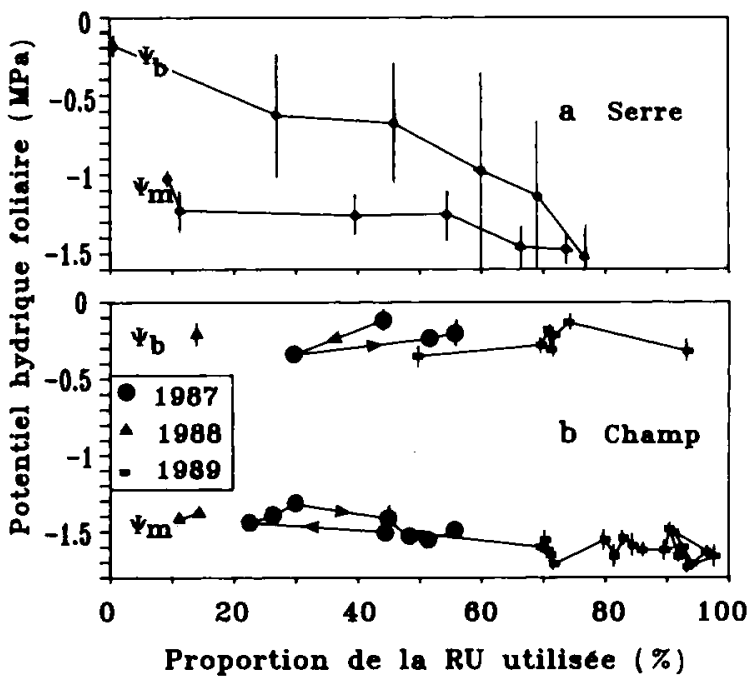

Fig 5. Potentiel hydrique foliaire mesuré à la fin de la nuit (base, $\left.\psi_{b}\right)$ et à midi solaire $\left(\psi_{m}\right)$, en fonction de la proportion de réserve en eau du sol utilisée par rapport à la réserve utile estimée. l'essai en pots, une gamme de réserve en eau de 0 à $100 \%$ a été parcourue en 9 jours. Une gamme plus faible a été parcourue chaque année dans l'essai au champ, mais la juxtaposition des 3 années correspond à une gamme de 13 à $98 \%$ de la $R U$. Seules ont été retenues dans cette relation les valeurs de $g_{s}$ maximales et de $\psi_{m}$ observées pour des éclairements supérieurs à $1 \mathrm{mmol}$ (quanta) $\cdot \mathrm{m}^{-2} \cdot \mathrm{s}^{-1}$.

Dans l'essai en pots, le potentiel de base a baissé jusqu'à $-1,5 \mathrm{MPa}$ pour un stock d'eau utilisé représentant $78 \%$ de la $R U$. En revanche, $\psi_{m}$ est resté quasiment stable sauf au tout début de l'expérience. La conductance stomatique a chuté à partir du troisième jour, pour un stock d'eau utilisé de $40 \%$ de la $R U$, ce qui correspondait à un $\psi_{b}$ de $-0,5 \mathrm{MPa}$ et un potentiel du sol de -0,3 MPa.

Au champ, ces indicateurs ont peu varié sur une large gamme de réserve d'eau du sol. Le potentiel de base est resté à des valeurs élevées $(-0,3 \mathrm{a}-0,2 \mathrm{MPa})$ en juillet et août 1989 , malgré le faible potentiel du sol (fig 4 ); $\psi_{m}$ a légèrement décru sur la gamme étudiée, passant de $-1,4$ à $-1,6 \mathrm{MPa}$. La conductance stomatique a varié entre 0,3 et $0,55 \mathrm{~cm} \cdot \mathrm{s}^{-1}$, indépendamment de la réserve d'eau du sol. On n'a donc pas observé de fermeture stomatique notable lors des 3 années d'étude.

Le nombre de grains par $\mathrm{m}^{2}$ a été maximal en 1989. Le poids moyen d'un grain (tableau II) n'a pas été significativement différent entre les 3 années d'étude, et était similaire à la moyenne obtenue pendant 8 ans (de 1982 à 1989) sur le même champ, et à celle obtenue sur une cam-

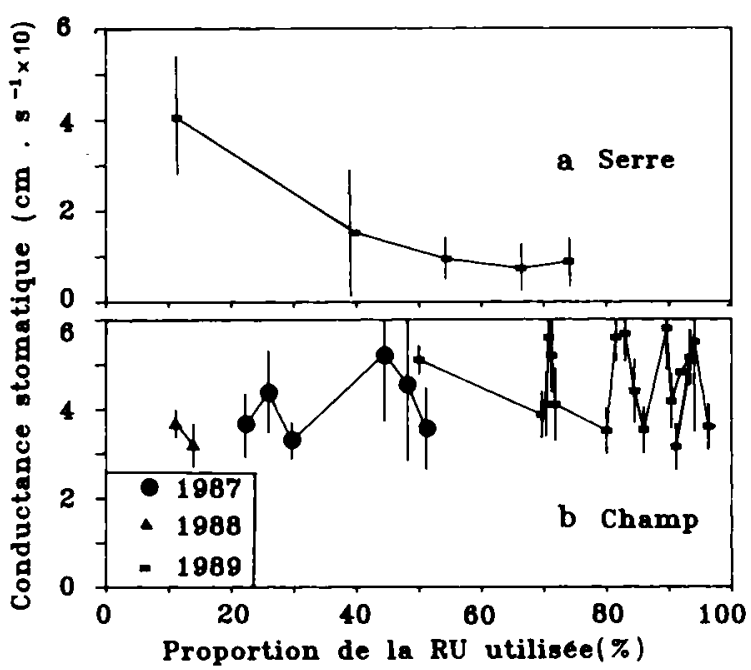

Fig 6. Conductance stomatique maximale journalière, mesurée avec des éclairements supérieurs à $1 \mathrm{mmol} \cdot \mathrm{m}^{-2} \cdot \mathrm{s}^{-1}$, en fonction de la proportion de réserve en eau du sol utilisée par rapport à la réserve utile estimée. 
Tableau II. Nombre de grains par $\mathrm{m}^{2}$ (ng) et poids moyen de matière sèche d'un grain (P1g) lors des 3 années d'étude, moyenne pour 8 ans dans la même parcelle et moyenne pour 5 ans dans 10 parcelles d'enquête pour la même variété (Hernandez et al, 1987). Les moyennes suivies par une lettre commune ne sont pas différentes suivant le test de Newman et Keuls, $p=0,05 ; x$ : moyenne; $s$ : écart type.

1987 1988 $1989 \begin{gathered}\text { Moyenne Moyenne } \\ 1982-1989 \text { enquête }\end{gathered}$

\begin{tabular}{lccccc}
$n g$ & & & & & \\
$x$ & $2178 \mathrm{~b}$ & $2606 \mathrm{a}$ & 2701 & 2331 & 2403 \\
$s$ & 260 & 108 & 219 & & \\
$P_{1 g}$ & & & & & \\
$x$ & $254 a$ & $264 a$ & $250 \mathrm{a}$ & 260 & 266 \\
$s$ & 14 & 11 & 18 & & \\
\hline
\end{tabular}

pagne de mesures concernant 10 parcelles pendant 5 ans (Hernandez et al, 1987).

\section{DISCUSSION}

\section{Relation potentiel du sol - potentiel foliaire}

Les valeurs minimales du potentiel foliaire observées pendant la journée ont peu réagi à l'état hydrique du sol. Ceci a été observé même dans l'essai en pots, alors que la conductance stomatique a décru avec le potentiel du sol. Cette stabilité de $\psi_{m}$ quel que soit l'état hydrique du sol et indépendamment de la conductance stomatique est conforme aux résultats obtenus pour le maïs par Dwyer et Stewart (1984) et Zhang et Davies (1989) en conditions contrôlées, et par Tardieu et al (1991) au champ. De l'apparente contradiction entre les mesures de potentiel foliaire et de conductance stomatique est née lidée que la conductance stomatique est contrôlée par un message chimique émis par les racines (Bates et Hall, 1981; Zhang et Davies, 1990; Tardieu et al, 1991).

Le potentiel de base dans l'essai en pots a suivi les variations du potentiel du sol. II lui était cependant toujours nettement inférieur, avec une différence de l'ordre de 0,8 MPa. Une telle différence a déjà été observée par Boyer (1974), et Neumann et al (1974), pour des plantes placées en solution nutritive. Elle est cependant plus grande dans cet essai, ce qui suggère que la ré- sistance au transfert d'eau entre le sol et les feuilles était trop grande pour que les plantes aient le temps de se mettre à l'équilibre avec le sol. Cela est vraisemblablement lié à la faible conductivité hydraulique de la tourbe et au mauvais contact entre celle-ci et les racines. Cette faible conductivité s'est également manifestée par le fait que les tensiomètres placés dans les pots n'ont pas réagi à la baisse de teneur en eau du substrat.

Le potentiel de base mesuré au champ a, au contraire, peu réagi aux variations de l'état hydrique du sol. De faibles flux d'eau parvenant aux racines les plus profondes ont été suffisants, en 1989 , pour que le potentiel des plantes atteigne un niveau élevé à la fin de la nuit. L'équilibrage du potentiel racinaire avec celui du sol s'est donc fait principalement dans le sens d'un transfert de l'eau du sol vers les racines. II est donc peu vraisemblable qu'un flux important d'eau ait quitté les racines vers le sol des couches les plus sèches, ce qui est conforme aux résultats de Dirksen et Raats (1985) sur luzerne. Des transferts de ce type sont, au contraire, probablement appréciables pour certaines plantes désertiques (Baker et Van Bavel, 1988; Caldwell et Richards, 1989).

Ces 2 expériences confirment donc des résultats d'autres équipes concernant la mauvaise relation entre l'état hydrique du sol et le potentiel hydrique foliaire mesuré pendant la journée. Elles montrent, de plus, que le potentiel de base peut être également assez mal relié au potentiel moyen du sol dans la zone enracinée, ainsi qu'aux variations de stock d'eau du sol. Elles montrent également que le potentiel de base est le résultat de transferts d'eau qui ne concernent pas l'ensemble du système racinaire, et qui ne sont pas toujours terminés à la fin de la nuit, comme ici dans l'expérience en pots.

\section{Apparition de la contrainte hydrique}

Dans l'essai en pots, la conductance stomatique $a$ été réduite à partir d'un potentiel hydrique du sol de $-0,3 \mathrm{MPa}$, qui a correspondu à un potentiel de base de $-0,5 \mathrm{MPa}$. Ces valeurs sont proches de celles trouvées par Dwyer et Stewart (1984). Si on définit la RFU comme la fraction de la $R U$ pour laquelle il n'y a pas de contrainte hydrique, le rapport RFU/RU était approximativement de 0,3 à 0,4 .

Dans l'essai au champ, les valeurs de la variation de la réserve d'eau du sol sont restées proches de celles du bilan ETP - $P$ lors des 3 
années, jusqu'à des valeurs de stock d'eau du sol correspondant à $50 \%$ de la $R U$ estimée (fig 4). Ceci suggère, d'une part, que l'évapotranspiration était alors proche de sa valeur maximale, peu différente de I'ETP Penman pour les $L A l$ atteints à la floraison, d'autre part que la mesure et le calcul du bilan hydrique du sol étaient satisfaisants tels qu'ils ont été menés. Le décalage entre le terme $(E T P-P)$ et la variation de stock d'eau du sol, observé pour des niveaux de $[\Delta S]^{2}$ d'environ $50 \%$ de la $R U$, aurait pu être interprété comme la conséquence d'une contrainte hydrique. Les mesures de potentiel de base et de conductance stomatique montrent au contraire l'absence de contrainte hydrique, puisqu'elles étaient à des niveaux similaires à ceux observés en sol peu desséché.

Le fort poids d'un grain en 1989 a été obtenu, bien que le nombre de grains par $\mathrm{m}^{2}$ ait été maximal cette année, probablement en raison du fort éclairement et des températures élevées pendant la période levée - floraison. Aucune des 2 composantes du rendement formées pendant la période que nous avons étudiée n'a donc été affectée en 1989 par les faibles potentiels hydriques du sol ni par le bilan hydrique.

Le calcul du bilan hydrique du sol et celui de la réserve utile se sont donc trouvés en défaut. Nos résultats ne remettent pas en cause la limite supérieure de dessèchement du sol par les plantes, couramment adoptée à $-1.6 \mathrm{MPa}$ (Gardner, 1988). II est à noter que la discussion sur la valeur de ce seuil de potentiel est sans grande portée pratique, puisque de fortes variations de seuil de potentiel se traduisent par de faibles variations de stock (fig 1 ), sauf pour des seuils quelque peu atypiques comme ceux déterminés par Maertens et al (1974). Les erreurs sur les variations de stock d'eau du sol proviennent surtout de la détermination du terme $D_{z}$, dont la valeur positive élevée en 1989 permet seule d'expliquer le bon comportement hydrique des plantes malgré la sécheresse. L'ordre de grandeur du flux $D_{z}$ obtenu dans l'expérience présentée ici, par application de la loi de Darcy ou par bilan hydrique, est compatible avec celui trouvé par Katerji et al, (1984) sur blé, par couplage du bilan d'énergie et du bilan hydrique.

\section{CONCLUSION}

Les 2 systèmes que nous avons étudiés se distinguent par le fait que l'état hydrique du sol était bien défini dans le cas de l'essai en pots (sys- tème semi-fermé), ce qui n'était pas le cas pour l'essai au champ (système ouvert).

En système semi-fermé, nous avons observé le cas classique pour le maïs : les valeurs journalières du potentiel foliaire mesuré pendant la journée ont peu varié dans le temps, alors que le potentiel de base et la conductance stomatique ont baissé avec le potentiel hydrique du sol. II semble possible, dans ce cas, de simplifier l'approche hydrodynamique des transferts d'eau par un bilan hydrique considérant une valeur seuil de $R F U / R U$, ou par la détermination de valeurs seuils du potentiel hydrique du sol. Cette conclusion serait encore probablement valide dans des parcelles où le sol peut être assimilé à un système semi-fermé à cause de la présence d'une couche de sol imperméable aux racines et à l'eau, et relativement proche de la surface.

En système ouvert, où les couches non enracinées peuvent fournir un flux d'eau important et mal connu, l'approche des contraintes hydriques par la seule caractérisation de l'état hydrique du sol s'est au contraire révélée inadaptée. Le potentiel de base et la conductance stomatique sont restés stables sur toute la gamme de variation du stock d'eau du sol, si celle-ci était considérée comme bornée supérieurement par la réserve utile calculée classiquement. Ils ont en particulier gardé des valeurs élevées en 1989, alors que le potentiel du sol dans la zone racinaire était en dehors de la gamme tensiométrique et que la réserve utile estimée était épuisée. Notons que l'analyse du rendement (nombre de grains et poids moyen d'un grain) a fourni une indication correcte de l'état des plantes dans ces conditions. Dans le cas présenté ici, l'erreur associée avec l'utilisation des critères «sol» a été commise dans le sens d'une sous-estimation de la capacité du sol à céder de l'eau au couvert végétal. Nous avons montré précédemment (Tardieu, 1987; Tardieu et al, 1991) que l'erreur commise va au contraire dans le sens d'une surestimation de cette capacité lorsque l'environnement des racines ne leur permet pas un fonctionnement optimal (tassement du sol, état sanitaire).

\section{RÉFÉRENCES}

Baker JM, Van Bavel CHM (1988) Water transfer through cotton plants connecting soil regions of differing water potential. Agron J 80, 993-997

Bates LM, Hall AE (1981) Stomatal closure with soil depletion not associated with changes in bulk leaf water status. Oecologia 50, 62-65 
Bethenod O, Jacob C, Rode JC, Morot-Gaudry JF (1982) Influence de l'âge sur les caractéristiques photosynthétiques de la feuille de maïs, Zea Mays $\mathrm{L}$ Agronomie 2, 159-166

Bethenod O, Katerji N, Quetin P, Bertolini JM (1988) Efficience de l'eau d'une culture de pomme de terre (Solanum tuberosum L cv Bintje). 1 Mise en évidence de la régulation du $\mathrm{CO}^{2}$ interne à l'échelle foliaire. Photosynthetica 22, 491-501

Bruckler L (1984) Utilisation de micropsychromètres pour la mesure du potentiel hydrique du sol en laboratoire et in situ. Agronomie 4, 172-182

Bruckler L, Lafolie F, Tardieu F (1991) Modelling the root water potential and soil-root water transport in the two-dimensional case. II Comparison with field experiments. Soil Sci Soc Am J à paraître

Boyer JS (1974) Water transport in plants : mechanisms of apparent changes in resistance during absorption . Planta 117, 187-207

Caldwell MM, Richards JH (1989) Hydraulic lift : water efflux from upper roots improves effectiveness of water uptake by deep roots. Oecologia 79, 1-5

Claasen MM, Shaw RH (1970) Water deficit effects on corn. II Grain components. Agron J 62, 652-655

Choisnel $E$ (1985) Un modèle agrométéorologique opérationnel de bilan hydrique utilisant les données climatiques. In “les besoins en eau des cultures" (Perrier A, Riou C, eds), pp 115-132. INRA, 920 pp.

Daynard TB, Duncan WG (1969) The black layer and grain maturity in corn. Crop Sci 9 (4), 473-476

Dirksen C, Raats PAC (1985) Water uptake and release by alfalfa roots. Agron J 77, 621-626

Dwyer LM, Stewart DW (1984) Indicators of water stress in corn. Can J Plant Sci 64, 537-546

Eagleman JR (1971) An experimental derived model for actual evapotranspiration. Agric Meteorol 8, 385394

Feddes RA, Kaba PJT, Bronswijk JJB, Halbertsma J (1988) Modelling soil water dynamics in the unsaturated zone. State-of-the-Art. J Hydrology (Amst) $100,69-111$

Gardner WR (1988) Soil properties and efficient water use, an overview. In : Limitations to efficient water use in crop production (Taylor HM, Jordan WR, Sinclair TR, eds). 45-64. ASA, Madison, USA 5, 38 p

Huguet JG (1985) Appréciation de l'état hydrique d'une plante à partir des variations micrométriques de la dimension des fruits ou des tiges au cours de la journée. Agronomie 5, 733-741

Hernandez A, Bloc D, Fleury A (1987) Compte rendu des essais croissance et développement du maïs. Publication «RNED céréales" \& "AIP Physiologie appliquée à la production du maïs". $104 p$

Jackson RD (1982) Canopy temperature and crop water stress. In : Advances in irrigation (Hillel D, ed) Academic press, New York, Vol. 1 pp 43-85

Jones HG (1980) Interaction and integration of adaptative responses to water stress : the implications of an unpredictable environment. In : Adaptation of Plants to Water and High Temperature Stress
(Turner NC, Kramer PJ eds). Wiley, New York, 353-365

Katerji N, Daudet FA, Valancogne C (1984) Contribution des réserves profondes du sol au bilan hydrique des cultures. Détermination et importance. Agronomie 7, 779-787

Katerii N, Itier B, Ferreira I (1988) Etude de quelques critères indicateurs de l'état hydrique d'une culture de tomate en région semi-aride. Agronomie 8, 425433

Klepper B, Browning VD, Taylor HM (1971) Stem diameter in relation to plant water status. Plant Physiol 48, 683-685

Maertens C, Blanchet R, Puech J (1974) Influence de différents régimes hydriques sur l'absorption d'eau et des éléments minéraux par les cultures. Régimes hydriques, systèmes racinaires et modalités d'alimentation en eau. Ann Agron (Paris) 25, 575586

Moltz FJ (1981) Models of water transport in the soilplant system : a review. Water Resour Res 6, 1346-1356

Mualem $Y$ (1976) A new model for predicting the hydraulic conductivity of unsaturated porous media. Water Resour Res 12, 513-521

Neuman HH, Thurtell GW, Stevenson KR (1974) In situ measurements of leaf water potential and resistance to water flow in corn, soybean and sunflower at several transpiration rates. Can J Plant Sci $54,175-184$

Perrier A, Archer P, Blanco de Pablos A (1974) Etude de l'évapotranspiration réelle et maximale de diverses cultures : dispositif et mesures. Ann Agron (Paris) 25, 697-731

Robelin M (1963) Contribution à l'étude du comportement du maïs grain vis à vis de la sécheresse. In : Journées internationales de l'irrigation, 69-76. AGPM, Paris

Smucker AJM, Mc Burney SL, Srivastava AK (1982) Quantitative separation of roots from compacted soil profiles by the hydropneumatic elutriation system. Agronomy J 74, 500-503

Tardieu F (1987) Etat structural, enracinement et alimentation hydrique du maïs. III Disponibilité des réserves en eau du sol. Agronomie 7, 279-288

Tardieu F (1988a) Analysis of spatial variability of maize root density. I. Effect of discontinuous wheel compactions on spatial arrangement of roots. Plant Soil 107, 259-266

Tardieu F (1988b) III. Effect of wheel compaction on water extraction. Plant Soil 109, 257-262

Tardieu F (1989) Root system response to soil structural properties : micro and macro scale. In : Mechanics and related processes in structured agricultural soils (Larson WE, et al eds) Kluwer Academic Publishers, 153-172

Tardieu F, Manichon H (1986) Caractérisation en tant que capteur d'eau de l'enracinement du maïs en parcelle cultivée. II. Une méthode d'étude de la répartition verticale et horizontale des racines. Agronomie $6,415-425$ 
Tardieu F, Katerji N, Bethenod O, Zhang J, Davies WJ (1991) Maize stomatal conductance in the field; its relationship with soil and plant water potentials, mechanical constraints and ABA content in the xylem sap. Plant Cell Environ (à paraître)

Van Genuchten MT (1980) A closed-form equation for predicting the hydraulic conductivity of unsaturated soils. Soil Sci Soc Am J 44, 892-896
Zhang J, Davies WJ (1989) Abscissic acid produced in dehydrating roots may enable the plant to measure the water status of the soil. Plant Cell Environ 12, 73-81

Zhang J, Davies WJ (1990) Changes in the concentration of $A B A$ in xylem sap as a function of changing soil water status can account for changes in leaf conductance and growth. Plant Cell Environ (à paraître) 